\title{
Pharmakomechanische Katheter-gesteuerte Thrombolyse: Keine Therapie der ersten Wahl bei TVT
}

Kearon Clive et al. Pharmacomechanical Catheter-Directed Thrombolysis in Acute Femoral-Popliteal Deep Vein Thrombosis: Analysis from a Stratified Randomized Trial. Thromb Haemost. 2019 Apr;119 (4):633-644

Die pharmakomechanische Kathetergesteuerte Thrombolyse (Pharmakomechanical catheter-directed thrombolysis, PCDT), die eine mechanische Thrombusdisruption in Kombination mit thrombolytischer Therapie beschreibt, entfernt den Thrombus sehr schnell und hat das Potenzial Kurz- und Langzeitoutcomes bei Patienten mit akuter tiefer Venen Thrombose (TVT) zu verbessern. In der ATTRACT-Studie konnte gezeigt werden, dass die Entfernung des Thrombus mittels PCDT nicht das postthrombotische Syndrom (PTS) als solches reduzierte, jedoch moderate bis schwere postthrombotische Syndrome und den Schweregrad der Symptome.

Nun wandten sich Kearon und Kollegen aus Hamilton, Ontario, der Frage zu, welchen Effekt die PCDT bei Patienten mit femoropoplitealer TVT hat (ohne Beteiligung der weiter proximal gelegenen Venen). Innerhalb der ATTRACT-Studie hatten $300 \mathrm{~Pa}$ tienten eine TVT mit Beteiligung der femoralen Venen ohne Beteiligung der V. fe- moralis communis oder der Vv. iliacae und wurden entweder in die Gruppe mit PCDT mit Antikoagulation oder in die Gruppe mit Antikoagulation alleine randomisiert. Die Patienten wurden 24 Monate nachverfolgt.

\section{Ergebnisse:}

Von 6 - 24 Monate gab es zwischen den beiden Gruppen keinen Unterschied, was das postthrombotischen Syndrom, das moderate oder schwere postthrombotische Syndrom, den Schweregrad des PTS, oder die allgemeine oder krankheitsspezifische Lebensqualität anbelangt ( $p>0,5$ für alle verglichenen Werte). Von 0 bis 10 und 30 Tage gab es keinen Unterschied bei der Verbesserung des Beinschmerzes oder der Schwellung zwischen den Behandlungsarmen. Von 0 bis 10 Tage traten in der PCDT-Gruppe bei 3 Patienten schwerwiegende Blutungen auf im Vergleich zu keiner Blutung $(P=0,006)$ in der Antikoagulationsgruppe und Blutungen generell bei 8 versus 2 Patienten $(p=0,032)$. Über 24 Monate kam es zu rekurrenten venösen Thrombembolien bei 16 der PCDT-Patienten und 12 der Patient ohne PCDT $(p=0,24)$.

\section{FAZIT}

Insgesamt zeigte sich in der ATTRACT-Studie, dass PCDT moderate oder schwerwiegende postthrombotische Syndrome reduziert, dass der Schweregrad des PTS reduziert und die Erholung von Beinschmerzen und Schwellung verbessert wird. In der aktuellen Studie konnten solche Vorteile bei Patienten mit femoropoplitealer TVT nicht gefunden werden. Da PCDT mit Blutungen assoziert ist, erscheint ihr früher Einsatz nicht indiziert bei Patienten mit femoropoplitealer TVT ohne Beteiligung der proximal liegenden Venen, so schließen die Autoren.

MOR Dr. med. Benedikt Lampl, Regensburg 